\title{
Searching User Generated Content: What's Next?
}

\author{
Maarten de Rijke \\ ISLA, University of Amsterdam \\ Kruislaan 403 \\ 1098 SJ Amsterdam \\ The Netherlands \\ mdr@science.uva.nl
}

\begin{abstract}
In recent years, blog search has received a lot of attention. Since the launch of dedicated evaluation efforts and the release of blog data sets, our understanding of blog search has deepened considerably. But there's more to user generated content than blogs and there's more to searching user generated content than looking for material that is relevant or opinionated or highly rated by readers or authors. In this talk, a number of user generated content search scenarios from the media analysis and intelligence domains will be detailed. From these, recurring themes such as credibility, people finding, impact prediction, unusual event detection, report and summary generation - all on user generated content-will be identified as highly relevant research directions.
\end{abstract}

